\title{
4-Dimensional Metrology Architecture for Satellite Clusters using Crosslinks
}

\author{
John W. Garnham \\ SAIC Corp. \\ Air Park Rd. \\ Albuquerque, NM \\ 505-842-7841 \\ john.w.garnham@saic.com \\ Frank R. Chavez; Thomas A. Lovell; \\ Air Force Research Lab/VSES \\ Kirtland AFB NM \\ 505-853-4132; 505-846-6066 \\ thomas.lovell@kirtland.af.mil; frank.chavez@kirtland.af.mil \\ Lynn M. Black \\ Air Force Research Lab/VSSS \\ Kirtland AFB NM \\ 505-846-5583 \\ lynn.black@kirtland.af.mil
}

\begin{abstract}
The interest ${ }^{1,2}$ in employing satellite clusters for multiple space-based missions typically requires significant cluster-level relative metrology. The metrology must be 4dimensional in general (3D space and time). A two-level metrology architecture and initial analysis using a combination of Carrier-Phase GPS measurements combined with inter-satellite crosslinks will be described. The crosslink approach generalizes the two-way time transfer methodology to n-way. This measurement approach simultaneously determines both separation and relative timing errors between spacecraft. As more satellites are added to the system, the number of additional independent measurements increases faster than the number of variables to be estimated thus improving performance and robustness.
\end{abstract}

\section{TABLE OF CONTENTS}

1. INTRODUCTION . .1

2. Motivation

3. Relative Metrology via Carrier Phase DIFFERENTIAL GPS (CDGPS) .2

4. Two-WaY and Three-WaY Time Transfer ..........3

5. THREE SATELLITE EXAMPLE ........................................

6. Relative Orbit Determination \& Prediction..5

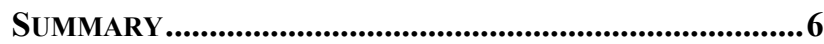

REFERENCES .....................................................................

BIOGRAPHIES ..................................................................

\footnotetext{
${ }^{1}$ U.S. Government work not protected by U.S. copyright

${ }^{2}$ IEEEAC paper\#1217, Version 5, Updated Dec 22, 2004
}

\section{INTRODUCTION}

The development of highly accurate relative metrology between free-flying spacecraft is essential for most satellite cluster applications. The satellites need to know where they are relative to each other, as well as predict where they will be with sufficient accuracy to determine when and where to make small-scale orbital adjustments that maintain cluster geometry within required parameters. In addition to determining relative spatial position, accurate relative time is also required. Range and time measurements are coupled when using signal time-of-flight and the speed of light to measure distance between satellites. Timing synchronization for inter-satellite communication and payload synchronization may have additional relative timing accuracy requirements over and above ranging requirements. A relative ranging technique that simultaneously solves for both range and time offsets between satellites is developed in this paper.

\section{Motivation}

The development of a crosslink based relative metrology system is envisioned to simultaneously determine 4D metrology (3D position and timing) between spacecraft at high accuracy. There are numerous papers on using GPS for relative navigation $[1,2,3,4,9]$ that achieve relative accuracies on the order of 10's of cm's and tens of ps. While this is impressive, if better accuracy is needed, then a crosslink-based approach may be used. A crosslink system of a satellite cluster can be considered a local "GPS" 
constellation for itself. The same techniques used for Carrier-Phase Differential GPS (CDGPS) can be applied with slight modifications to the cluster metrology using crosslinks. The local crosslinks can operate at higher bandwidth and frequency, to improve both ranging and timing accuracy over GPS, especially if they exploit carrier phase tracking. A ranging accuracy improvement on the order of $10 \mathrm{x}$ is straightforward. A 10x increase would require $10 \mathrm{x}$ bandwidth (e.g. $\sim 10-100 \mathrm{MHz}$ ) and if carrier phase tracking is used, moving from L-Band to Ku-Band. This is a straightforward crosslink.

\section{Relative Metrology via Carrier}

\section{Phase Differential GPS (CDGPS)}

A brief discussion on CDGPS is given here to introduce the metrology concepts, and relate the similarities and differences between CDGPS relative metrology and the proposed crosslink relative metrology. For absolute GPS location determination, it is assumed that the GPS satellite locations/orbits are known (generally a very good assumption, as the orbits are measured to high accuracy). The ranges between the receiver and the satellites are determined. With just the range information, the location of the receiver is known to be on a sphere centered on the satellite with radius of the measured range. By ranging to multiple satellites, separate receiver location spheres are determined, and the location of the receiver is the intersection of these spheres. This very brief discussion ignores the effects of errors due to noise, or system-level errors. The resolution of real-world errors is attained via a maximum likelihood approach (e.g. Least Squares) to state estimation, and additional constraints and/or extra measurements to create an over-determined problem. This also tends to improve the robustness of the estimate.

There have been a number of recent papers and research in the area of relative satellite metrology using CDGPS. JPL has actual software code (GYPSI/OASIS) to calculate both absolute and relative orbits using CDGPS [1-2]. Researchers at MIT are developing a microsatellite formation flying experiment. Part of their development has included hardware-in-the-loop experiments to develop the experimental hardware and software. Their research has shown relative accuracies of $2 \mathrm{~cm}$ position accuracy and 0.5 $\mathrm{mm} / \mathrm{s}$ velocity accuracy [3]. NASA's GRACE satellite pair is presently on-orbit and mapping the earth's gravity field to very fine accuracy. GRACE has demonstrated better than $2 \mathrm{~cm}$ relative position accuracy and $5-10 \mathrm{ps}$ relative timing accuracy [4]. GRACE uses both CDGPS and an intersatellite crosslink to achieve these numbers. GRACE's crosslink approach is different from the one to be presented in this paper.

The general theory and equations to calculate position and time in CDGPS is as follows. Differential GPS uses differential range measurements which are the difference between GPS calculated pseudo ranges for a specific satellite and those for a reference point (which may be another satellite). These measurements are defined as:

$$
\delta \rho_{i_{-} \text {meas }}=\left(\mathbf{r}_{\mathrm{mi}}-\mathbf{r}_{\mathrm{d}}\right) * \mu_{\mathrm{i}}+\delta_{\mathrm{i}}
$$

where:

$\delta \rho_{\mathrm{i} \text { meas }}$ is the measured differential pseudo range between the reference satellite and satellite " $i$ "

$\mathbf{r}_{\mathrm{d}}$ is the position vector of the reference receiver $\mathbf{r}_{\mathrm{mi}}$ is the position vector of the satellite "i" to be measured $\mu_{\mathrm{i}}$ is the unit vector from reference satellite to satellite " $i$ " $\delta_{\mathrm{i}}$ is the sum of differential measurement errors for satellite "i"

“*” denotes the dot product.

These differential pseudo range measurements enable the differential position to be determined between the reference satellite and the rest of the satellites of the cluster. This effectively cancels all of the correlated system errors in the relative position measurements of the satellite cluster. The specific approach of how these differential pseudo ranges are combined to calculate position and time is discussed at length in many textbooks and reports [10] on GPS and will not be discussed in this paper.

This same approach can also be used with differential carrier phase measurements. The carrier phase measurements unlike the psuedorange measurements are ambiguous, with the GPS wavelength approximately $19 \mathrm{~cm}$. Since carrier phase can be solved to a fraction of a degree, subject to achieving large signal SNR, very fine range determination is possible. The relevent equation $\mathrm{s}$ are of the form shown in equation (2).

$$
\delta \rho_{\mathrm{i}}=\left(\mathbf{r}_{\mathrm{mi}}-\mathbf{r}_{\mathrm{d}}\right) * \mu_{\mathrm{i}}+\mathrm{N}_{\mathrm{i}} * \lambda+\varepsilon_{\mathrm{i}}
$$

where:

$\delta \rho_{i}$ is the differential carrier phase for satellite " $i$ " $\mathrm{N}_{\mathrm{i}}$ is the integer phase ambiguity for satellite " $\mathrm{i}$ " $\varepsilon_{\mathrm{i}}$ is the sum of differential measurement errors

$\lambda$ is the GPS carrier wavelength

$\mathbf{r}_{\mathrm{d}}, \mathbf{r}_{\mathrm{mi}}, \boldsymbol{\mu}_{\mathrm{i}}$ are as defined for equation (1)

Just as before, these carrier phase pseudoranges can be used to solve for each satellite's position and time offset. But before that can be done, the carrier phase ambiguity must be solved for. There are three general solution classes used to solve for this ambiguity that can be used separately or jointly: search methods, filtering methods and geometrical methods. Most techniques take on the order of 10 minutes to fully resolve the carrier phase ambiguities typically using a combination of the three techniques. Once the phase ambiguity is resolved, very accurate relative ranging can be achieved with correspondingly accurate relative location and time synchronization, as documented in the previously mentioned references [1-4]. 


\section{Two-WAY And Three-Way TIME}

\section{Transfer}

The previous section describes the state of the art in satellite metrology using GPS. The approach must be modified to include intersatellite crosslinks to improve position and timing synchronization accuracy. Others have looked at the merger of separate ranging systems with GPS [11]. Unlike the case with GPS where we can assume that we accurately know the location of the GPS satellites that we are ranging to, when ranging between satellites in a cluster we do not know where they are. We must simultaneously solve for the location of all of the satellites in the cluster. We can obviously use the results from the previously described CDGPS techniques as a starting point, but then must improve on them.

An additional complexity of intersatellite ranging to resolve both position and timing synchronization accuracy is the coupling between spatial and temporal displacement measurements between satellites. The solution for measurement of intersatellite ranging assumes temporal synchronization, and the measurement of intersatellite time offsets assumes intersatellite range knowledge. The technique of two-way and three-way time transfer was developed to solve this problem [5-8]. The formulations described in these references are based on using GPS, but the approach will be generalized to satellite cluster crosslinks.

The basic technique uses GPS as a common reference between two clocks, allowing relative timing errors to be determined. If two users $\mathrm{A}$ and $\mathrm{B}$ determine their time using the same GPS satellites at the same time, we can form

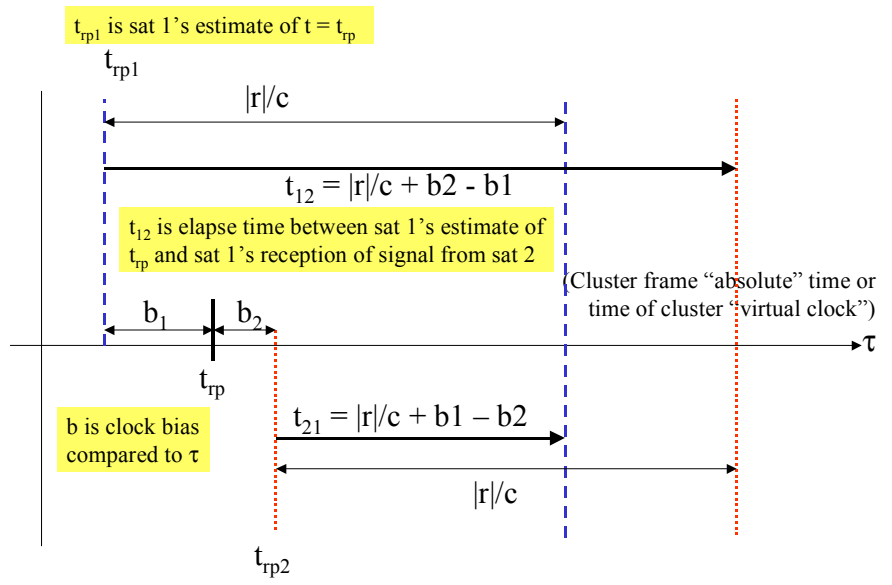

Figure 4-1 Two-way time transfer two separate equations as:

$$
\begin{aligned}
& \mathrm{A}=\mathrm{T}_{\mathrm{A}}-\text { GPS time } \\
& \mathrm{B}=\mathrm{T}_{\mathrm{B}}-\text { GPS time }
\end{aligned}
$$

Where A and B are the local clock GPS offset for users $A$ and $B, \mathrm{~T}_{\mathrm{A}}$ is the time of the local clock for user $\mathrm{A}$, and $\mathrm{T}_{\mathrm{B}}$ is the time of the local clock of user $\mathrm{B}$. The offset between user $A$ and $B$ 's clocks can be determined with the following equation that has the actual GPS time "cancel out."

$$
\mathrm{A}-\mathrm{B}=\left(\mathrm{T}_{\mathrm{A}}-\text { GPS time }\right)-\left(\mathrm{T}_{\mathrm{B}}-\text { GPS time }\right)=\mathrm{T}_{\mathrm{A}}-\mathrm{T}_{\mathrm{B}}
$$

The solution of equation (5) is the relative time difference between user clocks A and B. Obviously, what limits the accuracy of the time difference between clocks is the validity of the assumption that the GPS time for each receiver is the same. Measurement noise and uncorrelated system errors (e.g. hardware antenna and receiver differences) will cause these two GPS time estimates to be slightly different.

This description does not work for the case of the intersatellite crosslink problem where simultaneous solution of time difference and range must be solved. The approach is modified as follows (see figure 4-1). We will first look at the two-satellite case then extend the approach to $\mathrm{N}$ satellites. It is assumed that each satellite has a crosslink transceiver and that at predefined times each satellite will send out a "ranging pulse" in what each satellite believes is a simultaneous manner. The error in simultaneity is then calculated and this is used to calculate relative time errors and intersatellite range. The satellite 1 receive time of satellite 2's ranging pulse is $\operatorname{tr}_{12}$.

$$
\operatorname{tr}_{12}=\left(\left|\mathbf{r}_{12}\right| / c\right)+\left(b_{1}-b_{2}\right)+n_{1}
$$

where:

$\mathbf{r}_{12}$ is actual range between satellites 1 and 2

$\mathrm{b}_{1}$ is clock 1 bias compared to cluster "master" time

$\mathrm{b}_{2}$ is clock 2 bias compared to cluster "master" clock

$\mathrm{n}_{1}$ is time measurement noise

Similarly the receive time of satellite 2 of satellite 1's ranging pulse is:

$$
\operatorname{tr}_{21}=\left(\left|\mathbf{r}_{21}\right| / \mathrm{c}\right)+\left(\mathrm{b}_{1}-\mathrm{b}_{2}\right)+\mathrm{n}_{2}
$$

We have two equations and four unknowns. The number of unknowns can be decreased to two in the following manner. First we exploit the fact that $\left|r_{12}\right|=\left|r_{21}\right|$, which decreases the number of unknowns to three. Then we can define $b_{2}=0$. This implies that we are defining "cluster time" as the time determined by satellite 2's clock. We can calculate both the range and time bias using these two equations 


$$
\begin{array}{r}
-\mathrm{b}_{1}+\left(\mathrm{n}_{1}-\mathrm{n}_{2}\right) / 2=\left(\operatorname{tr}_{12}-\operatorname{tr}_{21}\right) / 2 \\
\mathbf{r}_{12}+\mathrm{c}\left(\mathrm{n}_{1}-\mathrm{n}_{2}\right) / 2=\left(\operatorname{tr}_{12}+\operatorname{tr}_{21}\right) * \mathrm{c} / 2
\end{array}
$$

This approach allows the simultaneous resolution of both intersatellite range and time offsets directly between a pair of satellites. This approach can be generalized to three or more satellites. For cases with more than three satellites, the problem becomes significantly over-determined, which should improve the conditioning of $\bar{T}$ and the overall robustness of the solution. We can reformulate equations (8-9) in vector form in the following manner.

$$
\begin{gathered}
\vec{m}=\bar{T} \vec{s}+\vec{n} \\
\vec{s}=\left(\bar{T}^{T} \bar{T}\right)^{-1} \bar{T}^{T} \vec{m}+\vec{n}\left(\bar{T}^{T} \bar{T}\right)^{-1} \bar{T}^{T}
\end{gathered}
$$

Where for three satellites,

$$
\vec{m}=\left[\begin{array}{c}
t r_{12} \\
t r_{21} \\
t r_{13} \\
t r_{31} \\
t r_{23} \\
t r_{32}
\end{array}\right] \vec{s}=\left[\begin{array}{c}
\left|r_{12}\right| / c \\
\left|r_{13}\right| / c \\
\left|r_{23}\right| / c \\
b_{1} \\
b_{2}
\end{array}\right] \vec{n}=\left[\begin{array}{c}
n_{1} \\
n_{2} \\
n_{3} \\
n_{4} \\
n_{5} \\
n_{6}
\end{array}\right]
$$

$$
\bar{T}=\left[\begin{array}{ccccc}
1 & 0 & 0 & 1 & -1 \\
1 & 0 & 0 & -1 & 1 \\
0 & 1 & 0 & 1 & 0 \\
0 & 1 & 0 & -1 & 0 \\
0 & 0 & 1 & 0 & 1 \\
0 & 0 & 1 & 0 & -1
\end{array}\right]
$$

$\bar{T}$ 's singular values are: $\{2.4495,1.4142,1.4142,1.4142$, $1.4142\}$

In order to assess Kalman Filter performance for relative orbit determination and prediction described in section 6 , determination of measurement errors is required. For the relative range measurement the measurement error is a combination of error due to noise, and limits of range resolution. We can see that the singular values of $\bar{T}$ show that it is full rank. The equation to determine system error covariance matrix can be calculated as shown in equations (11, 11a):

$$
\vec{a}=\vec{n}\left(\bar{T}^{T} \bar{T}\right)^{-1} \bar{T}^{T}
$$

$$
\bar{C}_{\sigma}=E\left[\vec{a} \vec{a}^{T}\right]
$$

The variance of noise $\mathrm{n}$ (in time units) is determined from a combination of clock phase noise and system range resolution. This is shown in equations $(12,12 \mathrm{a})$

$$
\begin{gathered}
\sigma_{n}=\left(\sigma_{\text {clock_noise }}{ }^{2}+\sigma_{\text {res_time }}{ }^{2}\right)^{1 / 2} \\
\sigma_{\text {res_time }}=\sigma_{r_{-} \text {res }} / c
\end{gathered}
$$

where:

$\sigma_{\mathrm{n}}$ is the crosslink time measurement noise variance

$\sigma_{\text {res_time }}$ is the crosslink time measurement error variance due to actual range resolution limits

$\sigma_{r_{-} \text {res }}$ is the range measurement variance error due to bandwidth and receiver noise

$\sigma_{\text {clock_noise }}$ is the phase noise of the clock

We have assumed that the system resolution errors are Gaussian-distributed with mean zero. The noise covariance matrix calculated in equation (11a) describes the effect of measurement noise on the overall estimate errors. The next section will demonstrate a specific three-satellite example using the approach described.

\section{Three SATEllite EXAMPle}

In this section an example with satellites are described and the results of Monte Carlo analysis to assess estimation errors due to noise are provided. The assumptions and simplifications used in this analysis will be explicitly described.

One of the first points of this analysis is that the intersatellite range values are defined between crosslink phase centers. The conversion from crosslink phase center location to satellite body or some other location on the satellite is not performed. One of the implications is that high quality calibration of the crosslink phase center location to satellite body must be performed to make use of the predicted ranging accuracy. To the degree that phase center motion of the crosslink is not perfectly calibrated, it can be treated as an additional low frequency noise source that is quasi-static for individual crosslink measurements but independent over long time scales.

Note that it is the intersatellite range, not intersatellite position that is determined. The conversion from intersatellite range to position is similar to the conversion from GPS pseudo-ranges to user position. The specifics of this conversion are not described in this paper. The general framework of this conversion is discussed in section 6 , and is expected to be the subject of future papers. 
The initial state "truth" vector for the three satellites is given as $\vec{S}$ and the measurement vector, including the effects of noise, is $\vec{m}$. In order to calculate the measurement vector, the equation for each measurement $t r_{x y}$ including noise is shown in equation (13)

$$
\begin{array}{r}
\vec{s}=\left[\begin{array}{c}
145.5 m / c \\
155.1 \mathrm{~m} / c \\
162.6 \mathrm{~m} / \mathrm{c} \\
3 \times 10^{-10} s \\
-2 \times 10^{-8} s
\end{array}\right]=\left[\begin{array}{c}
488.255 \times 10^{-9} \\
520.470 \times 10^{-9} \\
488.638 \times 10^{-9} \\
3 \times 10^{-10} \\
-2 \times 10^{-8}
\end{array}\right] \mathrm{S} \\
t r_{x y}=\left|r_{x y}\right| / c+\left(b_{x}-b_{y}\right)+n
\end{array}
$$

The variance of the noise in the Monte Carlo simulation is calculated based on range resolution and clock phase noise. It is assumed that the crosslink has a bandwidth of $100 \mathrm{MHz}$ and the system SNR is sufficient to resolve the modulated wavelength to $\lambda / 10$. This results in a range resolution of $298 \mathrm{~mm}$ and a temporal resolution of $1 \times 10^{-9} \mathrm{~s}$. The assumed clock phase noise is $1 \times 10^{-11} \mathrm{~s}$. The variance of the noise $\mathrm{n}$ is then calculated and used in the Monte Carlo simulation with 100,000 runs. The resulting state rms error is shown as

$$
\text { state_est_rms }=\left[\begin{array}{c}
704.823 \times 10^{-12} \\
706.182 \times 10^{-12} \\
704.543 \times 10^{-12} \\
576.528 \times 10^{-12} \\
577.652 \times 10^{-12}
\end{array}\right] s
$$

This result shows an improvement for both the range and time error variance as compared to a single measurement with an error variance of approximately $1 \times 10^{-9}$. The result is consistent with the SVD values of $\bar{T}$ using the following equations (14 and 15) to calculate the expected estimation variance for both range and time. We can see very good agreement between the predicted error variances and the calculated ones.

$$
\begin{gathered}
\sigma_{\text {final_r_error }}=\sigma_{t}\left\{\frac{1}{3}\left[3 \times\left(\frac{1}{1.4142}\right)^{2}\right]\right\}^{1 / 2} \\
\sigma_{\text {final_r_error }}=707.149 \times 10^{-12} s=.211 \mathrm{~m}
\end{gathered}
$$

$$
\begin{aligned}
& \sigma_{\text {final_t_error }}=\sigma_{t}\left\{\frac{1}{2}\left[\left(\frac{1}{2.45}\right)^{2}+\left(\frac{1}{1.4142}\right)^{2}\right]\right\}^{1 / 2} \\
& \sigma_{\text {final }{ }_{-}{ }_{\text {time }}{ }_{-} \text {error }}=577.38 \times 10^{-12} \mathrm{~s} \\
& \text { 6. RELATIVE ORBIT DETERMINATION \& } \\
& \text { PREDICTION }
\end{aligned}
$$

For the purpose of initial analysis, it is assumed that the inter-satellite separation behaves in a manner such that the relative orbital dynamics may be accurately described using linearized equations of motion, namely the ClohessyWiltshire (or Hill's) equations. This will allow for initial assessment of the potential for the proposed crosslink method to reduce relative position accuracies over current capabilities. In addition, requirements on the crosslink accuracy that guarantee reduced relative position accuracies can be established.

The Kalman Filter will be described for a three-satellite cluster but can easily be extended to an N-satellite cluster. Since the relative positions between the satellites is desired, the dynamics will be written in terms of the relative positions and velocities. Let the state of the entire threesatellite cluster system be expressed as follows:

$$
\mathbf{z}=\left\{\begin{array}{c}
z_{1} \\
z_{2} \\
\vdots \\
z_{18}
\end{array}\right\}=\left\{\begin{array}{l}
\overline{\mathbf{z}}_{1} \\
\overline{\mathbf{z}}_{2} \\
\overline{\mathbf{z}}_{3}
\end{array}\right\} ; \overline{\mathbf{z}}_{i}=\left\{\begin{array}{c}
x_{i} \\
y_{i} \\
z_{i} \\
u_{i} \\
v_{i} \\
w_{i}
\end{array}\right\}
$$

where $\left\{x_{i}, y_{i}, z_{i}\right\}$ and $\left\{u_{i}, v_{i}, w_{i}\right\}$ is the $\mathrm{i}^{\text {th }}$ satellite's position and velocity in the Hill's equation reference frame. The dynamics are linear and can be expressed in terms of the system state-transition matrix from time $t_{k}$ to the next update time, $\mathrm{t}_{\mathrm{k}+1}$ as

$$
\mathbf{z}_{k+1}=\mathbf{\Phi} \mathbf{z}_{k}
$$

The measurements to be used to update the Kalman Filter are the relative range estimates obtained from the crosslink method as described in Section 4 and 5. The output equations for the system dynamics is then given as 


$$
\mathbf{h}=\left\{\begin{array}{l}
h_{1} \\
h_{2} \\
h_{3}
\end{array}\right\}=\left\{\begin{array}{l}
\delta r_{12} \\
\delta r_{13} \\
\delta r_{23}
\end{array}\right\}
$$

with

$$
\begin{aligned}
& h_{1}=\sqrt{\left(x_{1}-x_{2}\right)^{2}+\left(y_{1}-y_{2}\right)^{2}+\left(z_{1}-z_{2}\right)^{2}} \\
& h_{2}=\sqrt{\left(x_{1}-x_{3}\right)^{2}+\left(y_{1}-y_{3}\right)^{2}+\left(z_{1}-z_{3}\right)^{2}} \\
& h_{3}=\sqrt{\left(x_{2}-x_{3}\right)^{2}+\left(y_{2}-y_{3}\right)^{2}+\left(z_{2}-z_{3}\right)^{2}}
\end{aligned}
$$

Let the state at update time $\mathrm{k}$ prior to the measurement update be denoted as $\mathbf{z}_{k}{ }^{(-)}$and the state at update time $\mathrm{k}$ just after the measurement update be denoted as $\mathbf{z}_{k}{ }^{(+)}$. Using this notation, the state dynamics estimate and the state measurement update sequence is given as

State Dynamics Estimate

$$
\begin{aligned}
& z_{k}^{(-)}=\Phi z_{k-1}^{(+)} \\
& P_{k}^{(-)}=\Phi P_{k-1}^{(+)} \Phi^{T}
\end{aligned}
$$

Calculation of Kalman gain

$$
\begin{gathered}
\mathbf{H}_{k}=\left[\frac{\partial \mathbf{h}}{\partial \mathbf{z}}\left(\mathbf{z}_{k}^{(-)}\right)\right] \\
\mathbf{K}_{k}=\left(\mathbf{P}_{k}{ }^{(-)} \mathbf{H}_{k}{ }^{T}\right)\left(\mathbf{H}_{k} \mathbf{P}_{k}^{(-)} \mathbf{H}_{k}{ }^{T}+\boldsymbol{\sigma}_{\delta r}{ }^{2}\right)
\end{gathered}
$$

State Update

$$
\begin{gathered}
\mathbf{z}_{k}^{(+)}=\mathbf{z}_{k}^{(-)}+\mathbf{K}_{k}\left[\mathbf{h}_{\text {crosslink }}-\mathbf{h}\left(\mathbf{z}_{k}^{(-)}\right)\right] \\
\mathbf{P}_{k}^{(+)}=\left(\mathbf{I}-\mathbf{K}_{k} \mathbf{H}_{k}\right) \mathbf{P}_{k}^{(-)}
\end{gathered}
$$

In the above equations, $\mathbf{P}$ is the state covariance matrix and $\sigma_{\delta \mathrm{r}}$ is the standard deviation of the resulting inter-satellite range estimates obtained from the crosslink method.

\section{SUMMARY}

This paper demonstrates how intersatellite crosslinks can be used to improve the relative position and time synchronization between satellites in a cluster formation. Future work will emphasize relative orbit determination performance and Filter "tuning" analysis. With crosslinks with sufficient and achievable performance, it would appear feasible based on this analysis to achieve at least a 10x improvement in relative navigation and timing over GPSonly techniques. A reasonable upper-bound approximation of the Kalman Filter performance over small time scales that the errors decrease by the square root of $\mathrm{N}$ for $\mathrm{N}$ measurements, thus sub-centimeter position and ps-level timing synchronization between spacecraft is possible. If we assume one measurement per second and 100 seconds of observation time, the range and time errors would decrease by 10 . This would result in ranging accuracies of $2.11 \mathrm{~cm}$ and timing accuracy of $58 \mathrm{ps.} \mathrm{Similarly,} \mathrm{for} 5400$ seconds ( 1 orbit period for a 90 minute LEO orbit), ranging error decreases to $.287 \mathrm{~cm}$ and $7.85 \mathrm{ps}$. 


\section{REFERENCES}

[1] B. Haines, W. Bertiger, S. Desai, D. Kuang, T. Munson, L. Young, P. Willis Jet Propulsion Laboratory, California Institute of Technology, Initial Orbit Determination Results for Jason-1: Towards a 1-cm Orbit, Proceedings, ION GPS-2002, Portland, OR.

http://gipsy.jpl.nasa.gov/igdg/papers/index.html

[2] R.J. Muellerschoen, A. Reichert, M. Heflin, W. Bertiger, Y. Bar-Sever, Orbit Determination with NASA's High Accuracy Real-Time Global Differential GPS System, Jet Propulsion Laboratory, Proceedings of ION GPS-2001, Salt Lake City, UT, September 2001.

http://gipsy.jpl.nasa.gov/igdg/papers/index.html

[3] J. How, F. Busse, Relative Navigation for Formation Flying Spacecraft Using Carrier-Phase Differential GPS. http://www.mit.edu/\%7Ejhow/gps1.htm

[4] W. Bertger, Y. Bar-Sever, S. Desai, C. Dunn, B. Haines, G. Kruizinga, D. Kuang, S. Nandi, L. Romans, M. Watkins, S. Wu, S. Bettadpur, GRACE: Millimeters and Microns in Orbit, Proceedings, ION GPS-2002, Portland, OR.

http://gipsy.jpl.nasa.gov/igdg/papers/index.html

[5] Dieter Kirchner, Two-way Time Transfer via Communication Satellites, Proceedings IEEE vol 79, No. 7, July 1991

[6] Lew, Wlodzimierz, Jacques, Andowski, Azoubib, and Klepczynski, GPS: Primary Tool for Time Transfer, Proceedings of the IEEE, Vol. 87, No. 1, January 1999

[7] K. Larson and J. Levine, Carrier-Phase Time Transfer, IEEE Transactions on Ultrasonics, Ferroelectronics, and Frequency Control, Vol 46, No. 4, July 1999

[8] E. Sappl, Comparison of a Ground and a Satellite Clock by Three-way Microwave Time Transfer, IEEE Transactions on Instrumentation and Measurement, Vol. 48, No. 4, August 1999

[9] A. Long, D. Kelbel, T. Lee, D. Leung, R. Carpenter, C. Gramling, Relative Navigation of Formation-Flying Satellites, presented at the NASA/CNES $1^{\text {st }}$ Annual Formation Flying Missions and Technologies International Symposium, Toulouse, France, Oct 29-31, 2002

[10] Stewart Cobb, GPS Pseudolites: Theory, Design, and Applications Doctoral Dissertation, Stanford University, 1997.

http://waas.stanford.edu/ wwu/papers/gps/PDF/stuthesis. pdf
[11] G. Opshaug, and P. Enge, Integrated GPS and UWB Navigation System Presented May 2002 at the IEEE UWB Science and Technology (UWBST) Conference, Baltimore, MD

http://waas.stanford.edu/ wwu/papers/gps/PDF/Opshaug UWBST02.pdf

\section{BIOGRAPHIES}

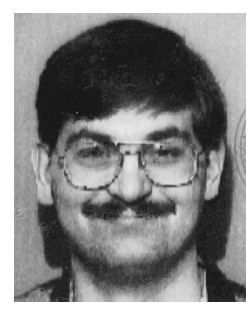

John Garnham is a Radar Systems Engineer at Science Applications International Corp (SAIC) on contract to the Air Force Research Laboratory (AFRL) at Kirtland AFB, $N M$ and Rome, NY. He is also a Flight Operations Systems Engineer on contract to Boeing Corp in El Segundo, CA. He has satellite operations, and ground support systems experience, along with surveillance technology strategic planning experience gained while serving as an Air Force officer and as an IPA at Phillips Laboratory (now AFRL). He is a Space Surveillance Concept Team leader of the Trilateral Technology Research and Development Program on Space Based Surveillance as an Air Force Reserve officer. $M r$. Garnham has a BS degree in Physics and Mathematics from Syracuse University, and completed satellite systems engineering technical training as an Air Force officer.

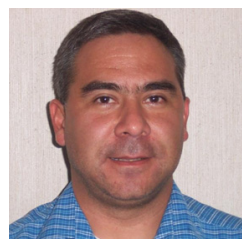

Dr. Frank R. Chavez is an Aerospace Research Engineer for the Air Force Research Laboratory Space Vehicles Directorate, Kirtland AFB, NM. As Team Lead for the Astrodynamics/Guidance, Navigation \& Control Team of the Simulation and Technology Assessment Branch (VSES), he is responsible for guiding research efforts and program support of team members. VSES provides support to the Integrated Flight Experiments Division in modeling and simulation of orbital and attitude dynamics of spacecraft systems. Dr. Chavez has expertise in satellite mission planning, ground operations, and flight dynamics and is an active member of AIAA and AAS. He has a Master's degree in Mechanical Engineering from Arizona State University and a Ph. D. in Aerospace Engineering from the University of Maryland. 


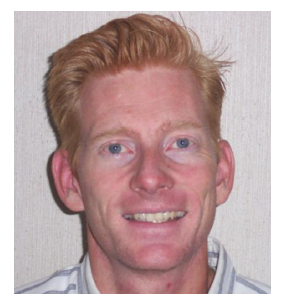

Dr. Thomas Alan Lovell is a member of the Astrodynamics/Guidance, Navigation, \& Control Team within the Simulation and Technology Assessment Branch of the Air Force Research Laboratory at Kirtland Air Force Base in Albuquerque, NM. His team provides support to the Integrated Flight Experiments Division in modeling and simulation of orbital and attitude motion of spacecraft. Dr. Lovell received a B.S. in Aerospace Engineering from Georgia Tech in 1991, an M.S. in Aerospace Engineering from Arizona State University in 1994, and a Ph.D. in Aerospace Engineering from Auburn University in 2001. He is an active member of AIAA and AAS, and is a member of the AIAA Astrodynamics Technical Committee and the AAS Spaceflight Mechanics Technical Committee. His research areas include: astrodynamics, orbit determination, trajectory optimization, and feedback control design. He has authored or co-authored over 10 journal articles and over 20 conference papers in these areas.

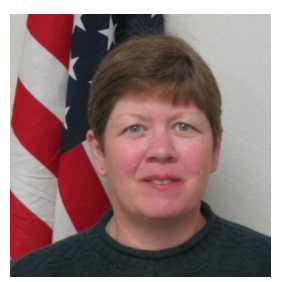

Ms. Lynn Black is the Active Sensors Group Lead and Program Manager for the Space-based Multi-Aperture Research and Technology program for the Air Force Research Laboratory Space Vehicles Directorate, Kirtland $A F B, N M$. She has experience in $R \& D$ flight test engineering (primarily infrared countermeasures systems), software engineering, and electronic combat systems. Ms. Black has managed research in nonlinear optics and lasers and imaging and has served as the Space Vehicles

Directorate Program Element Manager and Chief, Program Control. Ms. Black received a B.S. in Chemistry and B.A. in Mathematics from Kent State University, and a B.S. and M.S. in Electrical Engineering from the Air Force Institute of Technology. She received Defense Financial Manager certification in 2002. She is a member of the IEEE, the Association of Old Crows, and the American Society of Military Comptrollers. 